\title{
Investigation of Chirped InAs/InGaAlAs/InP Quantum Dash Lasers as Broadband Emitters
}

\author{
Mohammed Zahed Mustafa Khan, Tien Khee Ng, Member, IEEE, Chi-Sen Lee, \\ Pallab Bhattacharya, Fellow, IEEE, and Boon S. Ooi, Senior Member, IEEE
}

\begin{abstract}
In this paper, we assessed the effect of additionally broadened quantum dash (Qdash) optical transitions in the multi-stack dash-in-a-well laser structure at both, material and device level. A broad photoluminescence linewidth of $\sim 150 \mathrm{~nm}$ demonstrates the formation of highly inhomogeneous InAs-dashes across the stacks. The transmission electron microscopy revealed small (large) average dash height from the Qdash stack with thick (thin) over grown barrier layer. The Fabry-Perot laser diodes fabricated from this chirped structure exhibits unique device physics under the short pulsewidth (SPW) and quasi-continuous wave $(\mathrm{QCW})$ operation. Varying the ridgewidth $(W)$ from 2 to $4 \mu \mathrm{m}$ showed quenching of ultrabroad lasing signature in the SPW operation, and consistent even for a wide $15 \mu \mathrm{m}$ oxide strip laser diode. A lasing spectral split with reduced intensity gap in the center is observed in the QCW operation with the gap decreasing with increasing ridge-width. Such atypical lasing operation, influenced by the waveguiding mechanism is qualitatively realized by associating to the reduced vertical coupling effect of the Qdash stacks in the operation of small ridge-width lasers compared with large ridge-width and oxide stripe lasers, and leading to varying non-uniform distribution of carriers among the inhomogeneously broadened Qdash stacks in each case. Our chirped $2 \times 830 \mu \mathrm{m}$ ridge laser demonstrated marked improvement in the internal quantum efficiency $(\sim 80 \%)$ and $-3 \mathrm{~dB}$ lasing bandwidth, $>50 \mathrm{~nm}$ centered at $\sim 1.61 \mu \mathrm{m}$.
\end{abstract}

Index Terms-Broadband semiconductor laser, dash-in-well, InAs/InAlGaAs, InAs/InP, quantum dash, quantum dots, chirped active region, inhomogeneous broadening.

\section{INTRODUCTION}

M ANIPULATING the naturally occurring size or composition fluctuation in the self-assembled multi-stack quantum dot (Qdot)/Qdash has been an exciting topic of research for a decade, to achieve broad emission devices. Contrary to the conventional light emitting and laser diodes wherein the emission spectrum is generally narrowband,

Manuscript received September 22, 2013; revised November 12, 2013; accepted December 1, 2013. Date of publication December 5, 2013; date of current version December 17, 2013. The work was supported by the King Abdullah University of Science and Technology Competitive Research Grant CRG-1-2012-OOI-010.

M. Z. M. Khan, T. K. Ng, and B. S. Ooi are with the Photonics Laboratory, Computer, Electrical and Mathematical Science and Engineering Division, King Abdullah University of Science and Technology, Thuwal 23955, Saudi Arabia (e-mail: mohammedzahed.khan@kaust.edu.sa; tienkhee.ng@kaust.edu.sa; boon.ooi@kaust.edu.sa).

C.-S. Lee and P. Bhattacharya are with the Department of Electrical Engineering and Computer Science, University of Michigan, Ann Arbor, MI 48109 USA (e-mail: cslntuee1207@gmail.com; pkb@umich.edu).

Color versions of one or more of the figures in this paper are available online at http://ieeexplore.ieee.org.

Digital Object Identifier 10.1109/JQE.2013.2294092 a broad emission linewidth source covers many communication wavelength bands. This has been demonstrated by the self-assembled Qdot (Qdash) nano-structure devices in the $\mathrm{O}-(\mathrm{C}-\mathrm{L}-\mathrm{U})$ bands [1]-[4]. This wide spectrum generation is a stimulating technology finding applications in environmental monitoring (gas and bio sensors), metrology and spectroscopy, medical imaging [1], [2], [5], apart from their utilization as multiple wavelength source [6], comb source [7], in wavelength division multiplexed system, already demonstrated.

Two classes of semiconductor broadband sources are identified based on the region of operation, namely, superluminescent diode (SLD), working in the amplified spontaneous emission region, and the broad inter-subband and inter-band (referred to as broadband hereafter) laser diodes, working in the stimulated emission region. While the former laser type is based on cascaded active region design basically concentrating $>2 \mu \mathrm{m}$ infra-red regime [5], the latter laser type found notable attention, recently, because of their wavelength span of ground state transition in the optical communication wavelength window [1], [3]. As-grown InAs/GaAs (InAs/InP) ultrabroadband Qdot (Qdash) SLDs, spanning $-3 \mathrm{~dB}$ bandwidth of $\sim 80$ (110) $\mathrm{nm}$ has been reported with output power in few tens of $m W$ [2], [8], [9]. The thrust to achieve high power and broad bandwidth concurrently, which is rather difficult in SLDs, lead to the successful investigation of broadband Qdot/Qdash lasers by our group. We demonstrated a broad lasing $-3 \mathrm{~dB}$ bandwidth of $\sim 22 \mathrm{~nm}$ from both, multi-stack Qdot and Qdash lasers, with output powers in hundreds of $m W s$ [3], [4].

Lately, the drive to further enhance the spectral width of the self assembled Qdot/Qdash broadband sources advanced to the subsequent exploration of bandgap engineering of the active region. This is achieved either by deliberate increase of the dot/dash size distribution during growth (pre-growth bandgap engineering), normally termed as chirped active region, or by post growth bandgap engineering such as intermixing. In general, a substantially broadened SLD emission is reported spanning $>300 \mathrm{~nm}$ in the O-band by monolithic integration of multiple selectively intermixed active region [10]. We successfully established the capability of this technique under the stimulated emission regime by demonstrating enhanced lasing bandwidth of $\sim 41 \mathrm{~nm}$ from InAs/InP Qdash with improved device characteristics and emission in the C-band [11]. The other promising approach for chirping the emission energies of the Qdot/Qdash stacking layers in the multi-stack device structure is accomplished in various manners. For instance, 
varying the capping layer thickness or the spacer /barrier layer thickness [7], [12], [13], changing the amount of InAs deposition or conditions or both [14], [15], the position of the dot layer in the dot-in-well structure [16], hybrid Qdotquantum well (Qwell) [17]. In general, enhanced broad emission from both, SLDs $(\sim>100 \mathrm{~nm})$ and lasers $(\sim 75 \mathrm{~nm})$ have been reported, in addition to high performance characteristics observed in the latter case.

In this work, we explore the chirping technique in InAs/InGaAlAs/InP Qdash device structure with the aim of: (i) intentionally broadening the dash optical transitions across the four Qwell/Qdash stacks, and (ii) shed light on the Qdash laser device physics. The chirping is achieved by varying the InGaAlAs barrier layer thickness across the stacks which affects the vertical strain, thus leading to different amount of strain experienced by the overgrown Qdash layer and leading to dissimilar average ground state emission [18]. A broad stimulated emission of $>50 \mathrm{~nm}$ is achieved with improved device characteristics from the chirped Qdash laser structure which is $>20 \%$ enhancement compared to our previous reports. We addressed the Qdash device physics by comprehensively characterizing the laser device at two different pulsed current operations (SPW and QCW (long pulse width)), in addition to the geometrical effects such as the waveguiding mechanism. Our results show formation of low intensity gap in the QCW lasing spectrum (spectral split) of $W=2 \mu \mathrm{m}$ ridge case, considered as a decoupled Qdash system, and reduction in the SPW and QCW lasing bandwidth from $W$ $>4 \mu \mathrm{m}$ ridge/oxide-strip laser, indicating the formation of a coupled Qdash system. We make an attempt to explain these observations qualitatively.

\section{Material Characterization}

The material was grown by molecular beam epitaxy on (100) oriented n-type S-doped InP substrate. The active region is dash-in-an-asymmetric-well structure consisting of four stacks of InAs dashes embedded in InGaAlAs Qwell. The waveguide core is based on a standard undoped separate confinement heterostructure (SCH) of $200 \mathrm{~nm} \mathrm{In}_{0.52} \mathrm{Ga}_{0.28} \mathrm{Al}_{0.2} \mathrm{As}$ material, lattice matched to InP substrate. Each of the five monolayers (MLs) thick InAs dash layer is embedded within a $7.6 \mathrm{~nm}(1.3 \mathrm{~nm}$ lower thickness and $6.3 \mathrm{~nm}$ upper thickness $)$ thick compressively strained $\mathrm{In}_{0.64} \mathrm{Ga}_{0.16} \mathrm{Al}_{0.2}$ As Qwell separated by varying thickness $(20 \mathrm{~nm}, 15 \mathrm{~nm}, 10 \mathrm{~nm}$, and $10 \mathrm{~nm})$ tensile-strained $\operatorname{In}_{0.50} \mathrm{Ga}_{0.32} \mathrm{Al}_{0.18}$ As top barriers, starting from a $25 \mathrm{~nm}$ thick bottom barrier. The InAs Qdash layers were grown $1 \mathrm{ML}$ at a time, each separated by $5 \mathrm{~s}$ growth pauses. The chirped Qdash (CQD) device structure is completed by $1000 \mathrm{~nm}$ thick lower n-cladding of $\operatorname{In}_{0.52} \mathrm{Al}_{0.48} \mathrm{As}$ Si-doped layer, and $1900 \mathrm{~nm}$ thick $\mathrm{In}_{0.52} \mathrm{Al}_{0.48} \mathrm{As}$ upper p-cladding and $250 \mathrm{~nm}$ thick $\mathrm{In}_{0.53} \mathrm{Ga}_{0.47}$ As contact layers with Be-doping at concentrations of $2 \times 10^{18} \mathrm{~cm}^{-3}$ and $2 \times 10^{19} \mathrm{~cm}^{-3}$, respectively. Two partial structures were also grown for comparison purpose. In the first partial structure, termed as "fixed barrier Qdash" (FQD), the top barrier thickness of all the four stacking layers is fixed at $10 \mathrm{~nm}$. In the second partial structure, only single Qdash layer is grown, terminated by the top barrier layer, and named as "single Qdash" (SQD) structure. The material structures are characterized utilizing atomic force microscopy (AFM), transmission electron microscopy (TEM), and photoluminescence (PL) spectroscopy.

\section{A. AFM and TEM Studies}

Fig. 1(a) shows the AFM image of the Qdashes revealing the island formation with highly inhomogeneous dash sizes. The dashes are composed of mixture of wire-like and dotlike features and with dispersive heights. From various AFM images, the surface density of dashes is estimated to be $\sim 5 \times 10^{10} \mathrm{~cm}^{-2}$ with $\sim 70 \%$ coverage. The dash island formation is revealed by the cross section CQD TEM micrographs shown in Fig. 1(c), taken along the [011] (dash widths) and [0-11] (dash lengths) directions. Bright truncated pyramids within the Qwell are clearly visible in the [011] direction micrograph. The average dash width and length is estimated to be $\sim 18 \mathrm{~nm}$ and $\sim 100 \mathrm{~nm}$, respectively. Since, various experiments have shown that the dash/dot height has the pronounced effect on the ground state energy transition [19], we statistically estimated average dash height of each stacking layers from the TEM micrographs. The average dash height grown with thinnest barrier width $(\sim 3.0 \mathrm{~nm})$ is larger than intermediate $(\sim 2.7 \mathrm{~nm})$ and thickest $(\sim 2.5 \mathrm{~nm})$ barrier samples, with an error margin of $\pm 0.5 \mathrm{~nm}$. Although qualitative, our dash height measurements highlights that reducing the barrier thickness leads to an increased vertical strain coupling [15], [18], which results in red shift (increased average dash height) of the mean ground state emission of InAs Qdash stacks, in addition to the localized (in-plane inhomogeneity) energy dispersion within the Qdash stack. This is depicted in an illustrative energy band model of the CQD structure in Fig. 1(b). Note that size dispersion is one of the factor leading to enhanced inhomogeneity of the active region, not to forget the compositional variations also lead to dispersive dash optical transitions.

\section{B. Photoluminescence Study}

The PL measurements were carried out on all the three samples i.e. $\mathrm{CQD}, \mathrm{FQD}$ and $\mathrm{SQD}$ structures, at $77 \mathrm{~K}$, and with different excitation powers utilizing $1064 \mathrm{~nm}$ diode pumped solid state laser. Figs. 1(d)-(f) illustrate the power dependent PL spectra corresponding to the CQD, FQD, and SQD samples. The degree of dash height variation can be studied via the PL peak shift and the PL linewidth (measured at the full-width-half-maximum (FWHM)) with excitation density, as shown in Fig. 1(g). Note that the PL peak wavelength of the CQD and FQD samples coincides appreciably at both, low $\left(1.5 \mathrm{~W} / \mathrm{cm}^{2}\right)$ and high $\left(3000 \mathrm{~W} / \mathrm{cm}^{2}\right)$ excitation power densities, attaining values $\sim 1540 \mathrm{~nm}$ and $\sim 1460 \mathrm{~nm}$. Therefore, we attribute the first PL peak of the CQD to the emission from the $10 \mathrm{~nm}$ barrier Qdash stack ( $\mathrm{S}_{10}$ which refers to $\mathrm{S}_{10 \mathrm{a}}+\mathrm{S}_{10 \mathrm{~b}}$ ), as shown in Fig. 1(b). We postulate that $\mathrm{S}_{10 \mathrm{a}}$ and $S_{10 b}$ dashes contribute collectively as they correspond to identical barrier thickness, supported by various studies that show the barrier thickness has a pronounced effect on the dash emission energy (dash/dot formation), although, the TEM 
(a)

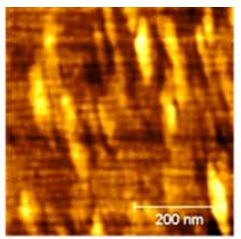

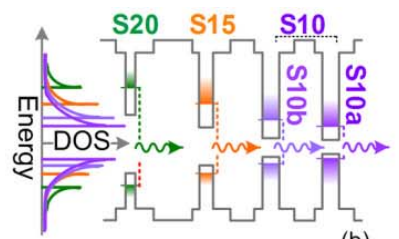

(b)

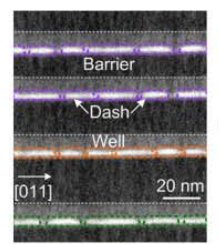

(c)

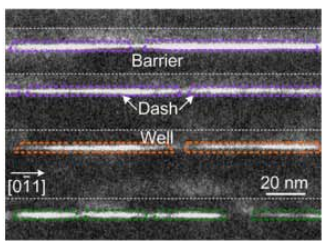

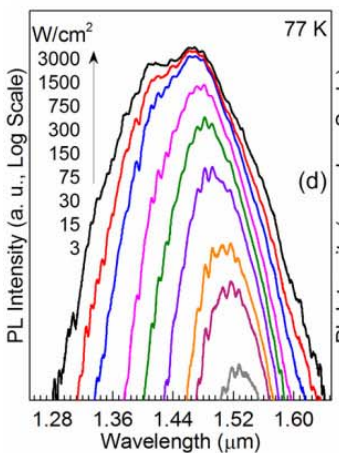
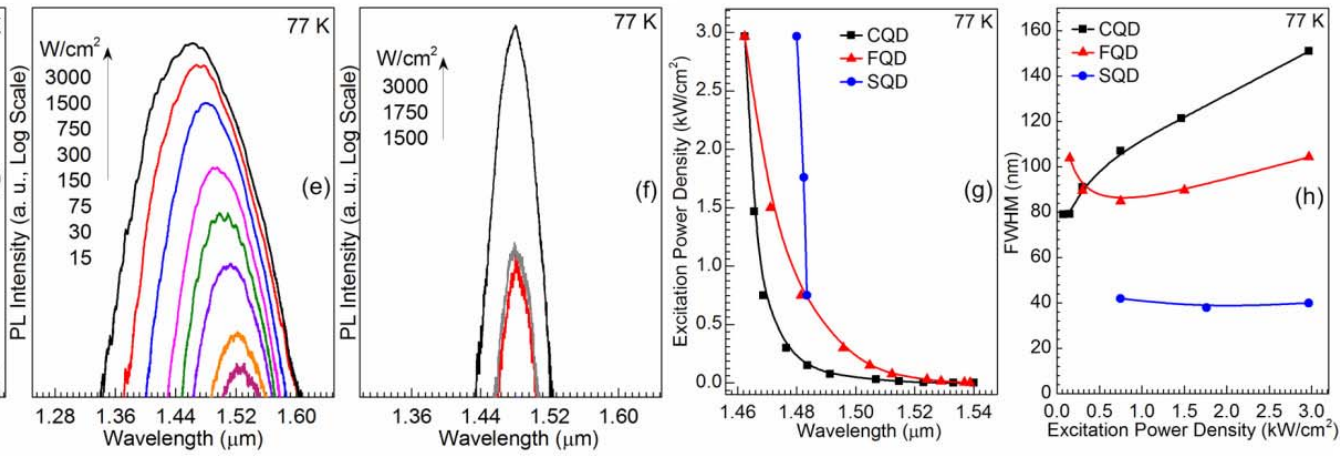

Fig. 1. (a) AFM image of Qdashes revealing the in-plane size dispersion. (b) Illustration of the energy band model of the CQD laser structure. (c) TEM images taken along [011] and [0-11] planes revealing the pyramidal type of the dashes with average width $18 \mathrm{~nm}$ and average length $100 \mathrm{~nm}$. Excitation power dependent 77K PL spectroscopy of the (d) CQD, (e) FQD, and (f) SQD structures. (g) Peak wavelength shift and (h) PL FWHM, as a function of the excitation power, for all the three samples. FQD and SQD sample results forms a basis for comparison purpose.

analysis shows different average dash height among these two stacks [15], [18]. Furthermore, these dash ensemble could be regarded as a coupled single dash ensemble system owing to the thin $10 \mathrm{~nm}$ barrier between the stacks which promotes vertical coupling, as has been observed in other reports [20]. The emerging second dominating PL peak at $\sim 1430 \mathrm{~nm}$ [Fig. 1(d)] suggests the noticeable emission from the $15 \mathrm{~nm}$ barrier Qdash layer $\left(\mathrm{S}_{15}\right)$. Emission from the short average height dashes is not evident from the PL spectra, however, a vague emission hump is observable at $\sim 1370 \mathrm{~nm}$ attributed to the emission from $20 \mathrm{~nm}$ barrier dashes $\left(S_{20}\right)$. We further performed Gaussian curve-fitting with the known PL peaks to identify the Gaussian peak corresponding to the short average height dashes. The results revealed the fourth Gaussian peak at $1360 \pm 10 \mathrm{~nm}$, which agrees well with our former observation. A broad PL linewidth (FWHM) [Fig. 1(h)] is noticed from CQD sample compared to the FQD sample, with increase in excitation power. At a high excitation of $3000 \mathrm{~W} / \mathrm{cm}^{2}$, $\sim 151 \mathrm{~nm}$ FWHM is obtained in the former case compared to $\sim 104 \mathrm{~nm}$ in the latter case, ascribed to the non-uniform distribution among largely inhomogeneous Qdash ensembles as a result of large degree of dash height variation across different layers of Qdash. In addition, small PL linewidth from the FQD suggests a vertically coupled Qdash system with reduced ground state energy distribution of the dash states, further supporting our earlier supposition.

To understand the effect of Qdash inhomogeneity on a single plane (in-plane or localized), we plotted the power dependent PL, PL peak shift, and FWHM of SQD sample in Fig. 1(f)-(h), respectively. The degree of PL peak blue shift with increasing excitation power which signifies, in this particular case, the presence of localized transition states from varied dimension dashes of the single stack, is minimal. The value attain a mere $4 \mathrm{~nm}$ compared to $>80 \mathrm{~nm}$ in CQD and FQD samples. In addition, the PL linewidth is relatively independent of excitation power (at $\sim 40 \mathrm{~nm}$ ). These observations indicate that in-plane inhomogeneity is not significant as compared to dash height variation across the Qdash stack, which shows that multi-stack active region is essential in achieving broad emission devices.

\section{Device Characterization and Discussion}

Ridge lasers (index-guided) with ridge-width $W=2,3$, and $4 \mu \mathrm{m}$, and oxide-strip lasers (gain guided) with stripewidth $W=15 \mu \mathrm{m}$, are fabricated with the standard fabrication process. The optical cavity of the lasers is aligned along [110] orientation and is perpendicular to the dash elongation direction to maximize the optical gain. After cleaving the sample into bars with different cavity lengths $(L=0.25-3.0 \mathrm{~mm})$, the non-facet coated lasers were tested on the temperaturecontrolled heat sink with an epitaxial p-side up mounting configuration. The testing at room temperature was performed under two different pulsed operation; SPW $(0.5 \mu$ s pulse width and $0.2 \%$ duty cycle), and QCW (100 $\mu$ s pulse width and $0.2 \%$ duty cycle) modes.

\section{A. Ridge-Waveguide Lasers}

In this section, we discuss the effect of two geometrical parameters of the index-guided lasers viz. the ridge-width and the cavity length, on the light power - current $(L-I)$ characteristics and the lasing operation, studied under both, the SPW and the QCW pulsed operations.

1) Effect of Ridge-width: Fig. 2 shows the effect of ridgewidth on the $L-I$ and emission characteristics of a ridge laser under the SPW operation. Here we fixed the cavity length to $L=1.6 \mathrm{~mm}$ to understand the lasing operation from the Qdash active medium at varying ridge-widths, although, all laser cavities show similar spectral behavior. From the $L-I$ characteristics, the threshold current density $\left(J_{t h}, \mathrm{kA} / \mathrm{cm}^{2}\right)$ 

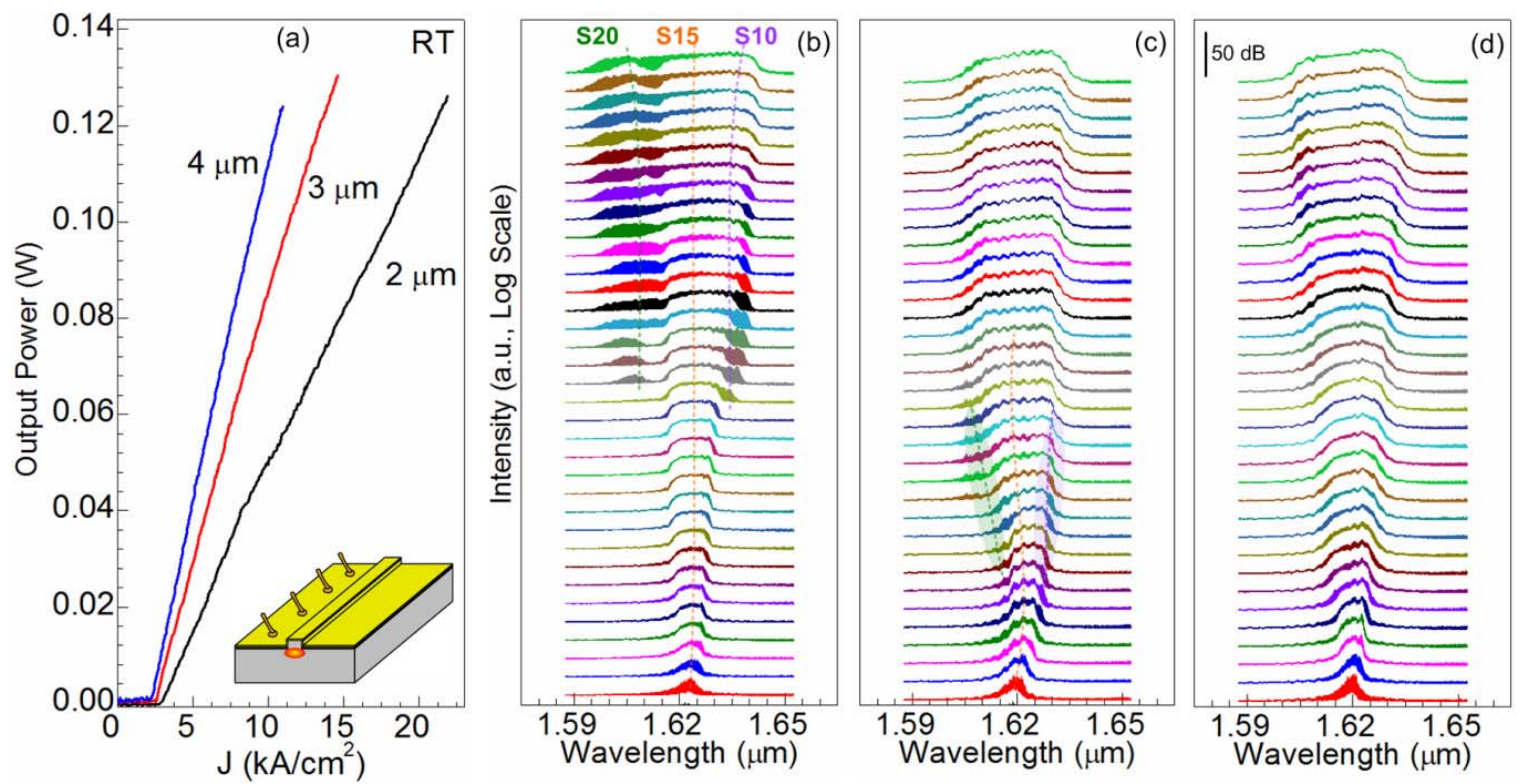

Fig. 2. (a) Room temperature $L-I$ characteristics of $L=1600 \mu m$ long CQD ridge laser at different ridge-widths, and in SPW operation. The corresponding progressive lasing spectra with increasing injection current density of (b) $W=2 \mu \mathrm{m}$, (c) $W=3 \mu \mathrm{m}$, and (d) $W=4 \mu \mathrm{m}$. In all the cases, the injection current density is varied from $1.0 \mathrm{~J}_{t h}$ to $4.5 \mathrm{~J}_{t h}$ in steps of $0.1 \mathrm{~J}_{t h}$. The dashed lines in (b) and (c) are guide to the eyes showing three dash groups dominating the lasing operation.

and slope efficiency (W/A) are $3.2(0.225), 2.7(0.234)$, and 2.3 (0.24), corresponding to $W=2,3$, and $4 \mu \mathrm{m}$ ridgewidth lasers. The extracted internal quantum efficiency $\left(\eta_{i}\right)$ and the internal loss $\left(\alpha_{i}\right)$ from the cavity length dependent characteristics, is observed to be consistent for all the ridgewidths, attaining values $\sim 80 \pm 3 \%$ and $11 \pm 1.0 \mathrm{~cm}^{-1}$, respectively. The increase (decrease) in $J_{t h}$ (slope efficiency) with decreasing ridge-width is attributed to the scattering loss at the side-walls (appreciable overlap of the optical mode and the side-walls) and lateral current spreading, which becomes significant in small ridge-width laser. In addition, in what follows, we partly ascribe the improved $L-I$ characteristics of large ridge-width lasers to the reduction in the inhomogeneous broadening of the active region. A weak kink observed in the $L-I$ curve of $W=2 \mu \mathrm{m}$ ridge laser, at around $\sim 2.7 \mathrm{~J}_{t h}$, while a comparatively smooth $L-I$ curves are observed in the other ridge-width lasers. This might be due to photon reabsorption in the small ridge-width laser as a result of highly inhomogeneous system. Besides, this is attributed to the lasing actions from different confined energy levels are not stable due to the occurrence of energy exchange between short and long wavelength Fabry-Perot lasing modes [21], spectra of Fig. 2(b) around that injection level.

Comparing the lasing spectra of different ridge-width lasers [Fig. 2(b)-(d)], a distinct lasing behavior is apparent from the short ridge-width laser. Typical broadening of lasing spectra is observed with increasing current pumping, however, at $\sim 2.7 \mathrm{~J}_{t h}$, lasing side lobes start to appear around the main spectral lobe, which broadens with further increase in injection, eventually coinciding with the main emission lobe and leading to a broad $-3 \mathrm{~dB}(-25 \mathrm{~dB})$ spectral bandwidth of $\sim 27 \sim 53) \mathrm{nm}$ [Fig. 2(b)]. This implies that three distinct dash ensembles, emitting at dissimilar wavelength in the stimulated emission regime, governs the lasing operation in this case. We attribute this to the three well decoupled Qdash stacks of the active region. Moreover, the threshold current of the spectral side lobes is around the similar current value where the kink in the $L-I$ curve was observed, further supporting our postulations of some photon re-absorption happening in the active region due to onset of lasing from other dash ensembles. We have explained the physics behind this broadband emission in greater detail later in this work. The lasing spectra of Fig. 2(c), representative of $W=3 \mu \mathrm{m}$ ridge laser show marginal such characteristics (highlighted by the shaded regions), but Fig. 2(d), which corresponds to $W=4 \mu m$ ridge laser, do not show such lasing behavior and instead a single spectral lobe is observed with increasing current pumping. The spectral broadening is marginal in both the cases, with $-3 \mathrm{~dB}$ bandwidth of $\sim 18 \mathrm{~nm}(W=3 \mu \mathrm{m})$ and $\sim 14 \mathrm{~nm}(W=4 \mu \mathrm{m})$. This is a signature of either an electronically coupled dash groups system where the dash ensembles emitting at different wavelength is hindered, or, a reasonably decoupled system with fairly different average ground state transitions of the Qdash ensembles. To further verify our postulation, we performed the current density dependent gain characteristics for all the three ridge-widths. The results are shown in Fig. 3, where the experimental dependence of the modal gain $\left(g_{m o d}\right)$ on the injection current density, $J$ (symbols), are obtained from different cavity lengths $(0.25-3.0 \mathrm{~mm})$. Fitting the data with the empirical gain expression (solid lines) [22], [23]:

$$
g_{\text {mod }}=g_{s a t}\left[1-\exp \left(-\gamma \frac{\left(J-J_{t r}\right)}{J_{t r}}\right)\right]
$$

revealed three gain curves for $W=2$ and $3 \mu m$ and two gain curves for $W=4 \mu \mathrm{m}$ ridge laser. The extracted transparency current density $J_{t r}$ and saturated modal gain, $g_{s a t}$ are shown in Table I for all the ridge-widths. Referring 

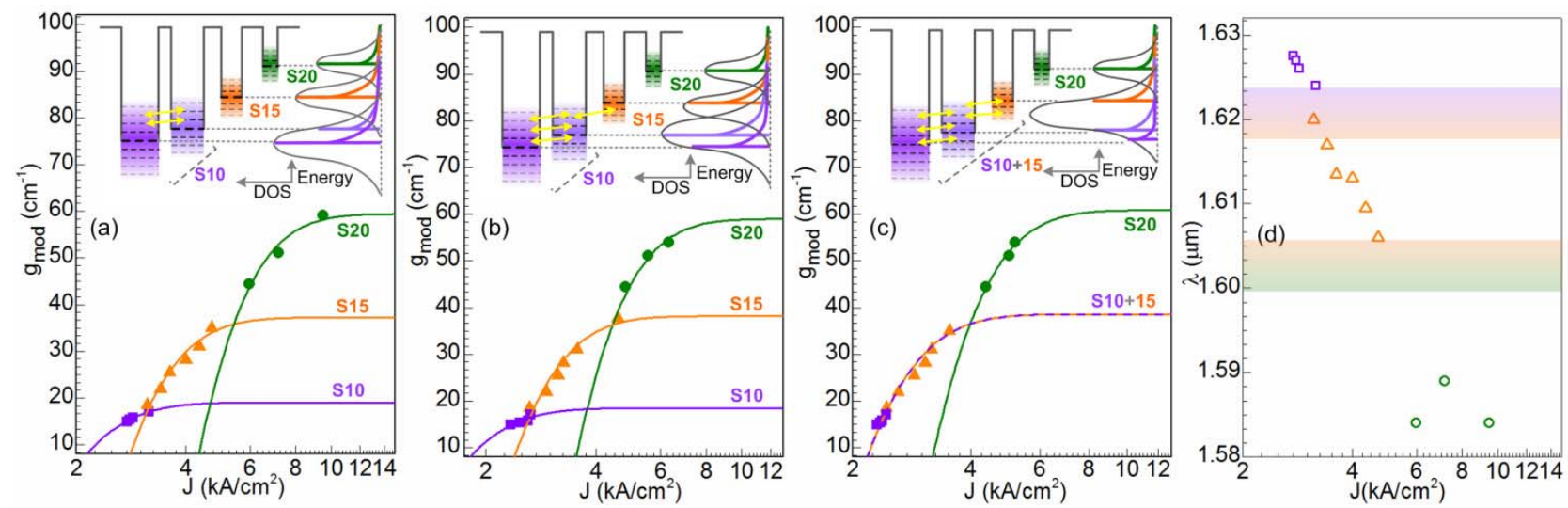

Fig. 3. Modal gain of CQD ridge lasers at (a) $W=2 \mu m$, (b) $W=3 \mu m$, and (c) $W=4 \mu m$ ridge-widths, versus the threshold current density. (d) Room temperature $1.1 \mathrm{~J}_{t h}$ lasing wavelength of $W=2 \mu \mathrm{m}$ ridge laser devices versus the threshold current density. The data is obtained at room temperature and from devices with different cavity lengths $(0.25-3.0 \mu \mathrm{m})$ in SPW operation The shaded region in (d) vaguely show the wavelength boundaries of the three dash ensembles. The solid lines in (a) - (c) are the theoretically fitted gain curves using Eq. (1), with non-ideality factor fixed at $\gamma=3$ and internal loss $\alpha_{i}=11 \mathrm{~cm}^{-1}$. The insets of (a) - (c) illustrates the conduction band model of the active region, describing the behavior of the Qdash ensembles under various ridge-widths. The width of the Gaussian curve of each Qdash stacks defines the in-plane inhomogeneity. Note the decrease in the inhomogeneous broadening of the active region (total Gaussian curves coverage of the system) with increase in the laser ridge-width, resulting from increased coupling effect (yellow arrows). Therefore, (a), (b), and (c) are considered as well decoupled, reasonably decoupled, and coupled Qdash system, respectively.

to Fig. 3(a), these gain curve are the characteristics of three dash ensembles emerging from $\mathrm{S}_{10}$ (upper), $\mathrm{S}_{15}$ (middle), and $\mathrm{S}_{20}$ (lower) stacks, in the $W=2 \mu \mathrm{m}$ case. Note that the absence of separate gain curves from $S_{10 a}$ and $S_{10 b}$ dash groups further supports our former attribution of these Qdash stacks working as a coupled single dash ensemble. We also show a qualitative wavelength coverage of these dash groups in Fig. 3(d) by plotting the near threshold lasing wavelength $(\lambda)$ of different laser cavities. The qualitative emission boundaries of these dash ensembles are shown as shaded regions with values $1608( \pm 6) \mathrm{nm}$ and $1622( \pm 3) \mathrm{nm}$. This analysis shows that the active region of $W=2 \mu \mathrm{m}$ ridge laser can be considered as a well decoupled Qdash system, thus, largely exploiting the intentional inhomogeneous broadening across the stacks. On the other hand, two gain curves in $W=4 \mu m$ ridge lasers, shown in Fig. 3(c), suggests reduced inhomogeneous broadening in this case. A collective single gain curve from $S_{10}$ and $S_{15}$ data points is indicative of another coupled $\mathrm{S}_{10+15}$ Qdash system which is consistent with increasing barrier thickness (i.e. $15 \mathrm{~nm}$, in this case) in the active region. Therefore, effect of collective contribution from the three Qdash stacks (coupled Qdash system) reduces the inhomogeneity of the active region, thus shrinking the gain spectrum, which fundamentally limits the laser emission bandwidth. In fact, similar behavior (two gain curves) is observed even in the gain-guided lasers, under the SPW operation, discussed later in this work, further strengthening our coupled Qdash system attribution. We ascribe this to the alteration in the active region temperature at high current injection, which depends on the geometrical parameters of the laser (ridge/stripe width in our case), and might accelerate the carrier tunneling process in the large ridge-width lasers, thus leading to reduced inhomogeneous broadening.

From the above analysis, the $W=3 \mu m$ ridge is regarded as a reasonably decoupled system with partial overlap of ground state energy distribution of each Qdash stacks compared to the negligible overlap in the $W=2 \mu \mathrm{m}$ ridge case, as illustrated in the inset of Fig. 3(b). As discussed before, the hazy appearance of spectral side lobes in Fig. 2(c) which persists for a short span of current pumping and then dominated by the progressive broadening of a single main emission lobe with further current injection, supports our attribution. In addition, these groups of longitudinal lasing modes which correspond to the different stacking layers are more clearly visible in the QCW mode [Fig. 4(b)] discussed later in this section. It is noteworthy to mention that the gain study of all the three ridgewidths and oxide-strip laser show consistent $g_{\text {sat }}$ values of the different Qdash ensembles i.e. $18 \pm 1.0 \mathrm{~cm}^{-1}\left(\mathrm{~S}_{10}\right), 38.5 \pm$ $1.5 \mathrm{~cm}^{-1}\left(\mathrm{~S}_{15}\right)$, and $59.5 \pm 0.5 \mathrm{~cm}^{-1}\left(\mathrm{~S}_{20}\right)$ thus showing the effectiveness of the analysis. The decrease (or early reach) of $J_{t r}$ of the Qdash stacks with increasing ridge-width points out to the early observation of the side lobes in the $W=3 \mu \mathrm{m}$ compared to $W=2 \mu \mathrm{m}$, on increasing current injection, that is exactly what Fig. 3(b) and(c) demonstrate.

Next, we show the consequence of changing the pulse width on the lasing operation of different ridge-width Qdash lasers. The results are plotted in Fig. 4 for all the three ridge-widths considered in this work and operating under QCW mode. A clear unusual lasing behavior is apparent on comparing with the SPW operation (Fig. 2). Moreover, the output optical power is substantially reduced in all the three ridge-width lasers suggesting enhanced loss mechanism in this case, partly attributed to junction heating. Indeed, the extracted $\eta_{i}(\sim 65$ $\pm 3 \%)$ and $\alpha_{i}\left(13 \pm 1.0 \mathrm{~cm}^{-1}\right)$ also points out to the device performance degradation in this operation. Our main aim to perform QCW investigation in this work is to understand the device physics when a highly inhomogeneous Qdash system is subjected to continuous surge of carriers, which probably would serve as guidelines to engineer and optimize the active region. Note that sharp abrupt kinks at $\sim 2.8 J_{t h}, \sim 4.0 J_{t h}$, and $\sim 5.5 J_{t h}$ in the $L-I$ of $W=2 \mu \mathrm{m}$ [Fig. 4(a)] case further dictates intense photon re-absorption around these injection levels . This is further demonstrated by observation of spectral split into two groups, short (SM) and long (LM) wavelength 

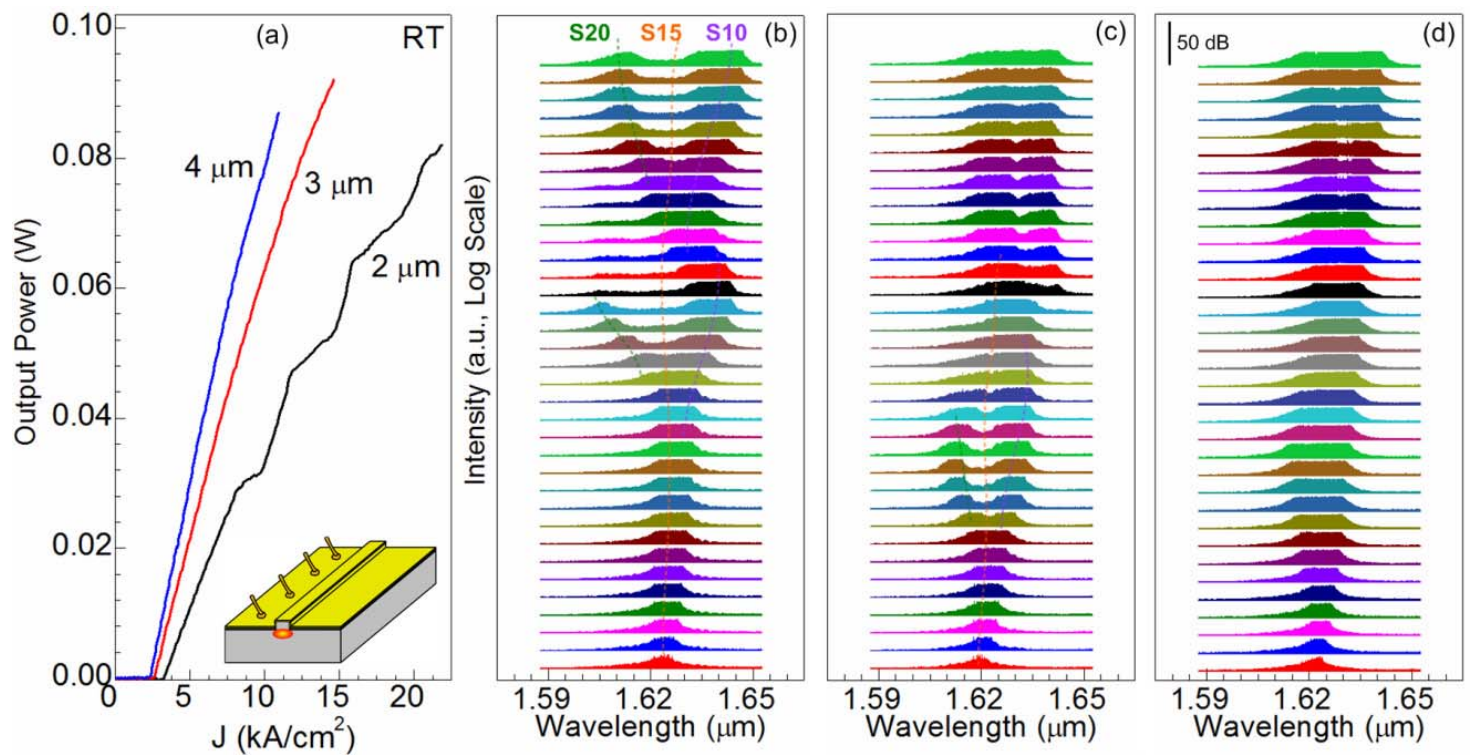

Fig. 4. (a) Room temperature $L-I$ characteristics of $L=1600 \mu \mathrm{m}$ long CQD index-guided laser at different ridge-widths, and in the QCW operation. The corresponding progressive lasing spectra with increasing injection current density; (b) $W=2 \mu \mathrm{m}$, (c) $W=3 \mu m$, and (d) $W=4 \mu \mathrm{m}$. In all the cases, the injection current density is varied from $1.0 J_{t h}$ to $4.5 J_{t h}$ in steps of $0.1 J_{t h}$. The dashed lines in (b) and (c) are guide to the eyes showing three dash groups dominating the lasing operation.

TABLE I

GAIN PARAMETERS EXTRACTED FROM THE EXPERIMENTAL DATA FOR THE THREE RIDGE-WIDTHS UNDER SPW OPERATION

\begin{tabular}{cccc}
\hline \hline \multirow{2}{*}{ Ridge-width } & Qdash group & $g_{s a t}\left(\mathrm{~cm}^{-1}\right)$ & $J_{t r}\left(\mathrm{kA} / \mathrm{cm}^{2}\right)$ \\
\hline \multirow{2}{*}{$2 \mu \mathrm{m}$} & $\mathrm{S}_{10}$ & 19 & 1.8 \\
& $\mathrm{~S}_{15}$ & 37 & 2.6 \\
\multirow{4}{*}{$3 \mu \mathrm{m}$} & $\mathrm{S}_{20}$ & 59.4 & 4.1 \\
& $\mathrm{~S}_{10}$ & 18.5 & 1.5 \\
& $\mathrm{~S}_{15}$ & 38 & 2.2 \\
$4 \mu \mathrm{m}$ & $\mathrm{S}_{20}$ & 59 & 3.4 \\
& - & - & - \\
& $\mathrm{S}_{10+\mathrm{S}_{15}}$ & 38.5 & 2 \\
& $\mathrm{~S}_{20}$ & 61 & 3.1 \\
\hline \hline
\end{tabular}

longitudinal modes, in conjunction with a reduced intensity group of middle (MW) wavelength modes in between, thereby forming a gap and eye-pattern like lasing spectra around the same injection levels, as depicted in Fig. 4(b). This atypical spectral behavior again dictates three dash groups governing the lasing operation in the QCW mode [15]. In fact, we studied the cavity length dependent gain characteristics of all the three ridge-widths, in this pulsed operation also, which resulted in an identical behavior of dash groups in all the three cases. The SW, MW, and LW group of longitudinal modes are the characteristics (being resonant with) of $\mathrm{S}_{20}, \mathrm{~S}_{15}$, and $\mathrm{S}_{10}$ Qdash stacks, respectively. We explained this anomalous observation in greater detail by considering a qualitative carrier-feeding model, very recently [24]. In the following, we briefly address the cause of this spectral split from the angle of highly inhomogeneous Qdash system, although, we cannot exclude the possibility of different non-linear phenomena occurring in the active region that affects the carrier-photon interaction, particularly, spectral and spatial hole burning effects, and the retarded carrier diffusion (due to potential fluctuations in the Qdash active region) and photon re-absorption (including the outer part of the ridge-waveguide) induced change in lateral index step and lateral gain distribution allowing higher order lateral mode to emerge [25]-[27]. All the above contributions usually intensify at high current injections and increased device heating.

Referring to Fig. 4(b), the lasing is initiated at $\sim 1614 \mathrm{~nm}$ corresponding to the $S_{15}$ dash groups and a typical broadening of lasing spectra is observed on subsequent injection. This is ascribed to the simultaneous lasing of dispersive in-plane $S_{15}$ dashes, and partly from adjacent $S_{20}$ stack. However, between $\sim 2.7 J_{t h}$ to $\sim 3.1 J_{t h}$ current pumping (large surge of carriers), quenching of $\mathrm{S}_{15}$ dashes (MW modes) results in a spectral split thereby recognizing the lasing SW $\left(\mathrm{S}_{20}\right)$ and LW $\left(\mathrm{S}_{10}\right)$ group of longitudinal modes. The formation and widening of the gap, i.e. reduction in the quantum efficiency of $S_{15}$ dashes, is a result of enhanced carrier feeding (via optical pumping) to $S_{10}$ dashes which are consequently attaining population inversion and lasing in tandem [see inset of Fig. 3(a)]. The blue (red) shifting of SW (LW) longitudinal modes is regarded as a result of localized broadening of the $\mathrm{S}_{20}\left(\mathrm{~S}_{10}\right)$ dash ensemble. Furthermore, sharp kink with a decrease in the slope of $L-I$ curve around the same current values is a signature of intense photon re-absorption process occurring in the system, which is due to the photon-carrier coupling between different dash groups as determined by the active region inhomogeneity. In the high injection regime $\left(\sim 3.2 J_{t h}\right.$ to $3.9 J_{t h}$ ), broadening and blue shifting of LW modes with reduced intensity from SW lasing modes is observed. This dictates increasing quantum efficiency of $S_{15}$ dashes and shift of carrier-feeding mechanism to $S_{20}$ dash ensemble. In other words, the single lobe spectra in this region is a collective lasing from $S_{15}$, in part, and $S_{10}$ dashes rather than only $\mathrm{S}_{10}$ dashes. The rise in the slope of $L-I$ curve highlights minimized absorption process in the region due to shift to 

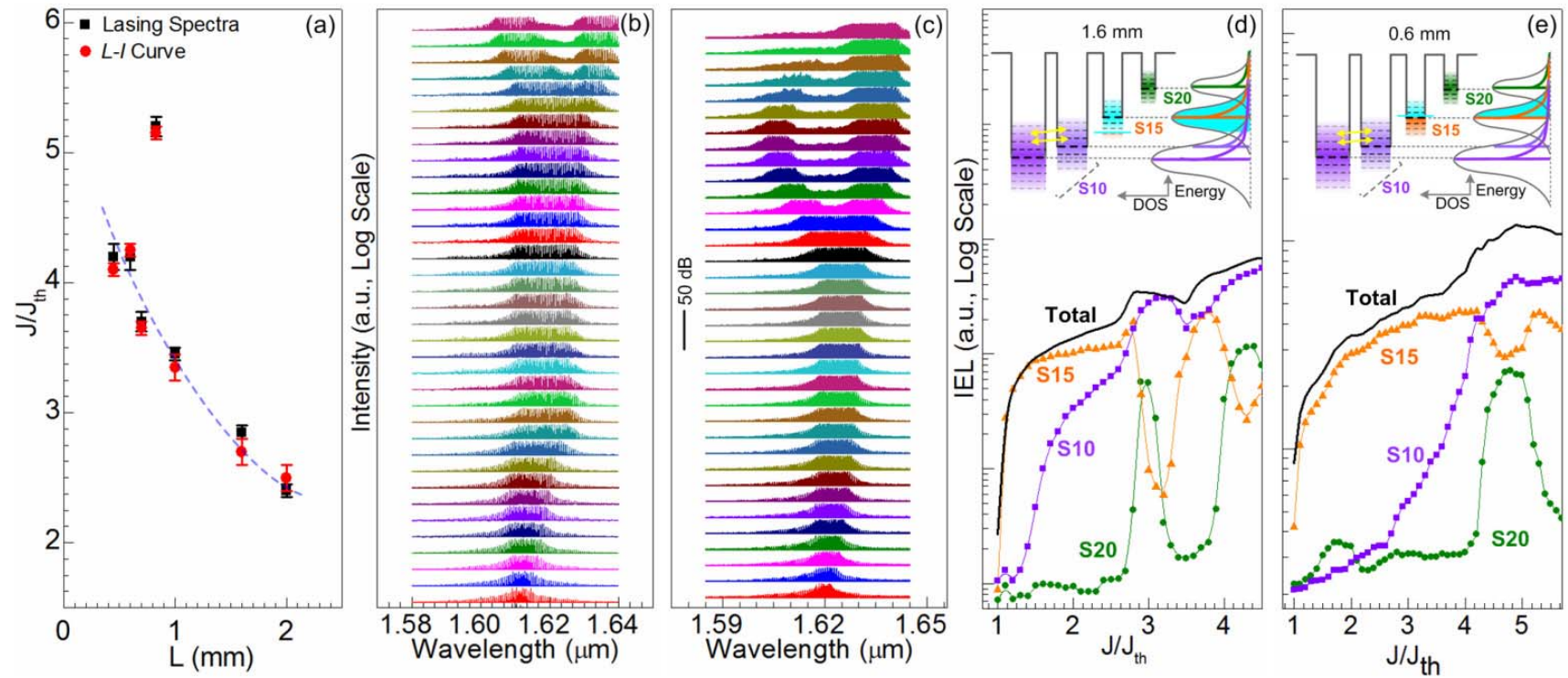

Fig. 5. (a) Cavity length dependent observation of first kink (red symbols, obtained from the respective $L-I$ curves), and the first spectral split in the lasing spectra (black symbols, from the current dependent lasing spectrum) of CQD $W=2 \mu \mathrm{m}$ ridge laser under QCW operation. The progressive lasing spectra with increasing injection current density for the short (b) $L=0.6 \mathrm{~mm}$ and intermediate (c) $L=1.1 \mathrm{~mm}$ cavity length lasers. Log IEL of the lasing spectra versus the current injection, for the long (d) $L=1.6 \mathrm{~mm}$ and short (e) $L=0.6 \mathrm{~mm}$ cavity lasers under QCW operation. The insets of (d) and (e) illustrates the conduction band model and filling of the $\mathrm{S}_{15}$ dash states (cyan color) in the different cavity lengths. The dashed line in (a) are guide to the eyes showing the trend. In all the cases, the injection current density is varied from $1.0 J_{t h}$ to $4.6 J_{t h}$ in steps of $0.1 J_{t h}$.

$\mathrm{S}_{20}$ dashes which are usually dot-like in nature with lower modal gain and density of states (DOS), hence fewer photon generation/absorption mechanism. Note that further increase in current pumping splits the single emission lobe and forms an eye-like pattern yet again, suggesting a periodic behavior, as shown in Fig. 4(b). This is possible in a highly inhomogeneous system due to change in carrier distribution among the Qdash stacks (caused by carrier spill-over) which affects the gain profile, and hence the emission spectra [21], [28].

A similar lasing characteristics is observed in $W=3 \mu \mathrm{m}$ ridge laser, as shown in Fig. 4(c). The spectral split forming an eye-like spectrum is weak in this case but the group of SW, MW, and LW longitudinal modes are noticeable. Moreover, a comparatively narrow spectral split wavelength coverage further uphold our attribution of $W=3 \mu \mathrm{m}$ active region as a reasonably decoupled Qdash system. A hazily periodic behavior with fairly single emission lobe at high injections $\left(>3.1 J_{t h}\right)$ highlights simultaneous emission from all the dash ensembles. Moreover, a smooth $L-I$ curve in this case is a consequence of reduced photon re-absorption process in the system. This is a direct effect of comparatively less inhomogeneous system compared to $W=2 \mu m$ case [29]. On the other hand, the lasing spectrum of $W=4 \mu \mathrm{m}$ laser show no signs of spectral split and instead a typical broadening of single emission lobe is observed. This is a signature of highly coupled Qdash system wherein the emission from different dash ensembles is indistinguishable (highly overlapping DOS of different dash ensembles). Comparing the lasing spectra in Fig. 4(b)-(d) again show an early spectral split on increasing the ridge-width which is consistent with our former observation in the SPW operation, and attributed to the reduced $J_{t r}$ value of the dash ensembles with increasing ridge-width.
From the above investigation, we ascribe QCW pulsed operation as a carrier deficient system for $W=2$ and $3 \mu \mathrm{m}$ ridge lasers because of various carrier-photon losses associated with it, for instance, non-radiation recombination, lattice vibrations, thermal carrier spill-over, which intensifies with temperature and/or continuous carrier injection, apart from the strong photon re-absorption process among the dash ensembles [30]. This increases the probability of dash ensemble quenching in QCW operation due to lack of evenly maintaining the rate of photon loss to the rate of photons generated, in the highly inhomogeneous multi-stack Qdash system, as a result of insufficient carriers. However, a single lobe emission from the $W=4 \mu m$ is suggests QCW mode being a carrier sufficient system in this case, since the photon re-absorption process is minimal (direct consequence of couple Qdash system) in spite of other loss mechanism occurring in the system. Alternatively, we regard the SPW mode as a carrier sufficient system for all the three ridge-widths since trivial photon re-absorption takes place in the system, besides minimal thermal carrier spill-over. Observation of a reasonably single lasing emission lobe at high current injection, in all the three ridge-widths (Fig. 2), suggesting an appreciable overlap of ground state emissions of all the dash ensembles (lasing simultaneously), further shows that SPW mode a carrier sufficient system.

2) Effect of Cavity Length: Fig. 5 shows the cavity length effect of $W=2 \mu \mathrm{m}$ ridge laser under the QCW pulsed operation. Comparing Figs. 4(b) and 5(b) and (c), which correspond to $L=0.6,1.1$, and $1.6 \mathrm{~mm}$, show similar observation of spectra splitting under stimulated emission, with increasing current pumping. This shows analogues nature of the well decoupled Qdash stacks of the short ridge-width active region, invariant of the laser cavity length. However, an earlier 

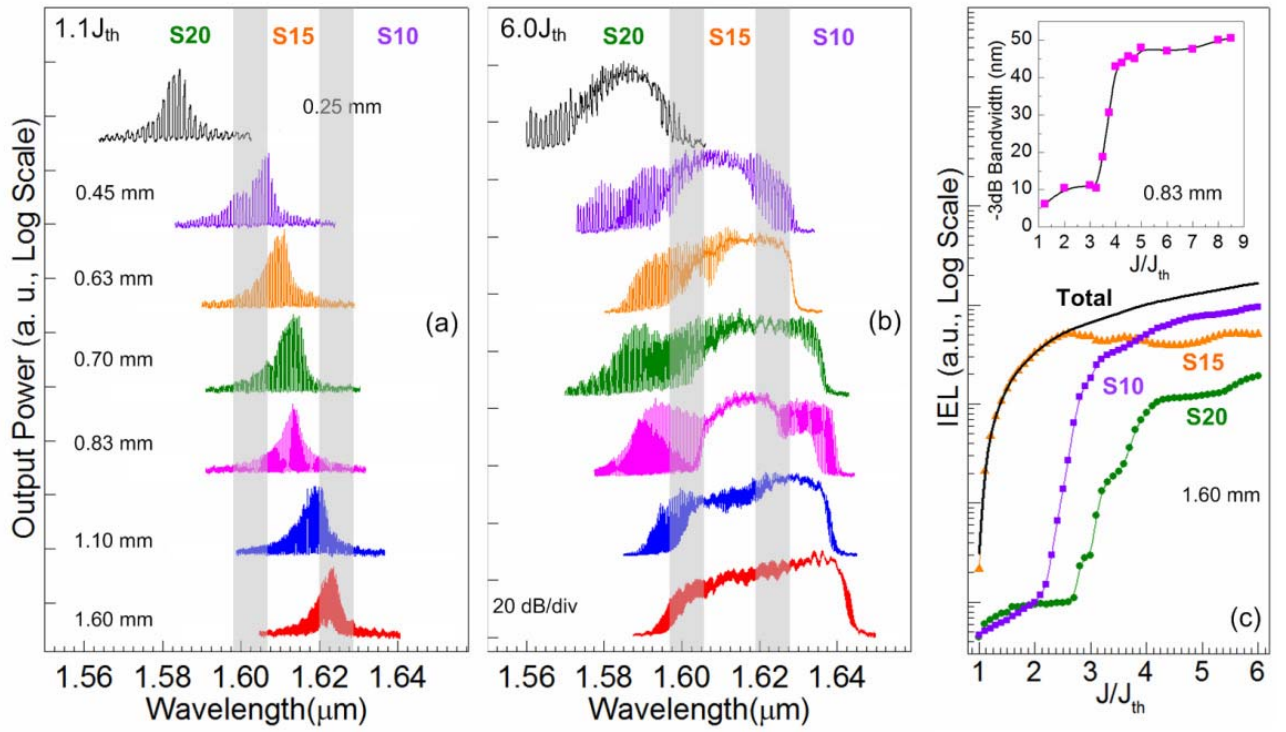

Fig. 6. Effect of cavity length under SPW operation, on the lasing spectra of the CQD $W=2 \mu m$ ridge laser under low (a) $1.1 J_{t h}$, and high (b) $6.0 J_{t h}$ current injection. (c) Log IEL of the lasing spectra versus the current injection for the long $L=1.6 \mathrm{~mm}$ cavity laser. The shaded regions in (a) and (b) correspond to the emission boundaries of the dash ensembles, obtained from Fig. 3(d). The inset of (c) shows the current density dependent change in the $-3 \mathrm{~dB}$ lasing bandwidth of a $2 \times 830 \mu \mathrm{m}$ long laser under SPW operation. An ultra broadband lasing bandwidth of $>50 \mathrm{~nm}$ is obtained from this cavity length laser.

occurrence of spectra splitting in $L=1.6 \mathrm{~mm}$ (at $\left.\sim 2.7 \mathrm{~J}_{t h}\right)$ is observed compared to the shorter $L=1.1 \mathrm{~mm}$ (at $\left.\sim 3.4 J_{t h}\right)$ and $L=0.6 \mathrm{~mm}$ (at $\sim 4.2 \mathrm{Jth}$ ) cavity lasers. This is plotted in Fig. 5(a) against various cavity lengths. In addition, the current values from the $L-I$ curve, at which the first kink is observed, is also shown in Fig. 5(a). A good agreement between both the current values (from the lasing spectra and $L-I$ curve) further support the cause of decrease in the slope efficiency being the spectral split due to enhanced photon re-absorption in the active region. We qualitatively explain the decrease in the threshold of spectral split with increasing cavity length by correlating to the active region inhomogeneity and the quasizero dimensional DOS of dashes, and referring to Fig. 5(d) and (e). Here, we plotted the integrated electroluminescence (IEL) of the three dash groups following the wavelength boundaries of dash ensembles discussed earlier, for the short $(L=0.6 \mathrm{~mm})$ and long $(L=1.6 \mathrm{~mm})$ cavity lasers. The onset of lasing from the long cavity laser is at $\sim 1620 \mathrm{~nm}$ i.e. from the extreme lower energy transition edge of $\mathrm{S}_{15}$ dash ensemble, implying that the photon re-absorption process has already started. In other words, the generated photons by the higher transition energy $S_{15}$ dashes [shown as cyan color $S_{15}$ dashes in Fig. 5(d)] are being absorbed by the dominant $S_{10}$ dashes. This is recognized from the early lasing threshold $\left(\sim 1.6 J_{t h}\right)$ of $\mathrm{S}_{10}$ dashes in Fig. 5(d) since they reach population inversion faster in this case. Therefore, feeding consequently emerging $\mathrm{S}_{10}$ dashes and lasing simultaneously, probably, results in an early gain saturation of $\mathrm{S}_{15}$ dash group which leads to quenching of their emission intensity, on subsequent injection. This can be seen in Fig. 5(d) as a relatively constant IEL curve of $\mathrm{S}_{15}$ dash stack starting from $\sim 2.7 J_{t h}$, the point where gain saturation is nearly reached, and eventually quenching because of inability to maintain the rate of photon generation and loss due to inherent DOS characteristics. In the case of short cavity device $(L=0.6 \mathrm{~mm})$, the lasing is initiated from the central transition energy of $S_{15}$ dash stack $(\sim 1613 \mathrm{~nm})$ which leaves more number of lower transition energy $S_{15}$ dashes to attain population inversion first while feeding the $\mathrm{S}_{10}$ dash group [shown as cyan color $\mathrm{S}_{15}$ dashes in Fig. 5(e)]. Therefore, the lasing threshold of $\mathrm{S}_{10}$ dash group is observed at comparatively large current value $\left(\sim 3.0 J_{t h}\right)$ in Fig. 5(e) once nearly entire $S_{15}$ dash ensemble simultaneously recombine in the system. Eventually, $S_{15}$ attaining gain saturation (and subsequently quenching), in this case, is at comparatively at large pumping values (at $>4.0 J_{t h}$ ) once enough $\mathrm{S}_{10}$ dashes reach population inversion. Note that the IEL of Fig. 5(e) is not accurate since the device heating is appreciable in this case compared to $L=1.6 \mathrm{~mm}$ case. The objective of showing Fig. 5(e) is just to explain the device physics qualitatively. In general, the inhomogeneous broadening of the system, DOS of dashes, and the carrier feeding (via optical pumping) mechanism determines this anomalous behavior of the lasing spectra in QCW operation.

We have shown that $W=2 \mu m$ ridge lasers probably exploits the entire inhomogeneity across the Qdash stacks under the SPW operation. However, dependence of the lasing operation on the cavity length (optical loss) cannot be disregarded. This can be noticed from Fig. 6 wherein the cavity length dependent lasing spectra at low $\left(1.1 J_{t h}\right)$ and high $\left(6.0 J_{t h}\right)$ current injections are plotted. Onset of lasing threshold from different dash ensembles [Fig. 6(a)] and a broad spectral coverage $(-25 \mathrm{~dB}$ bandwidth) of $>60 \mathrm{~nm}$ [Fig. 6(b), and for $L>0.36 \mathrm{~mm}$ ] is apparent for various cavity lengths. Notice that the broadest lasing linewidth $(-3 \mathrm{~dB}$ bandwidth) appears from the intermediate cavity length lasers $(L=0.83$ and $0.7 \mathrm{~mm}$ ) whose lasing threshold originate 

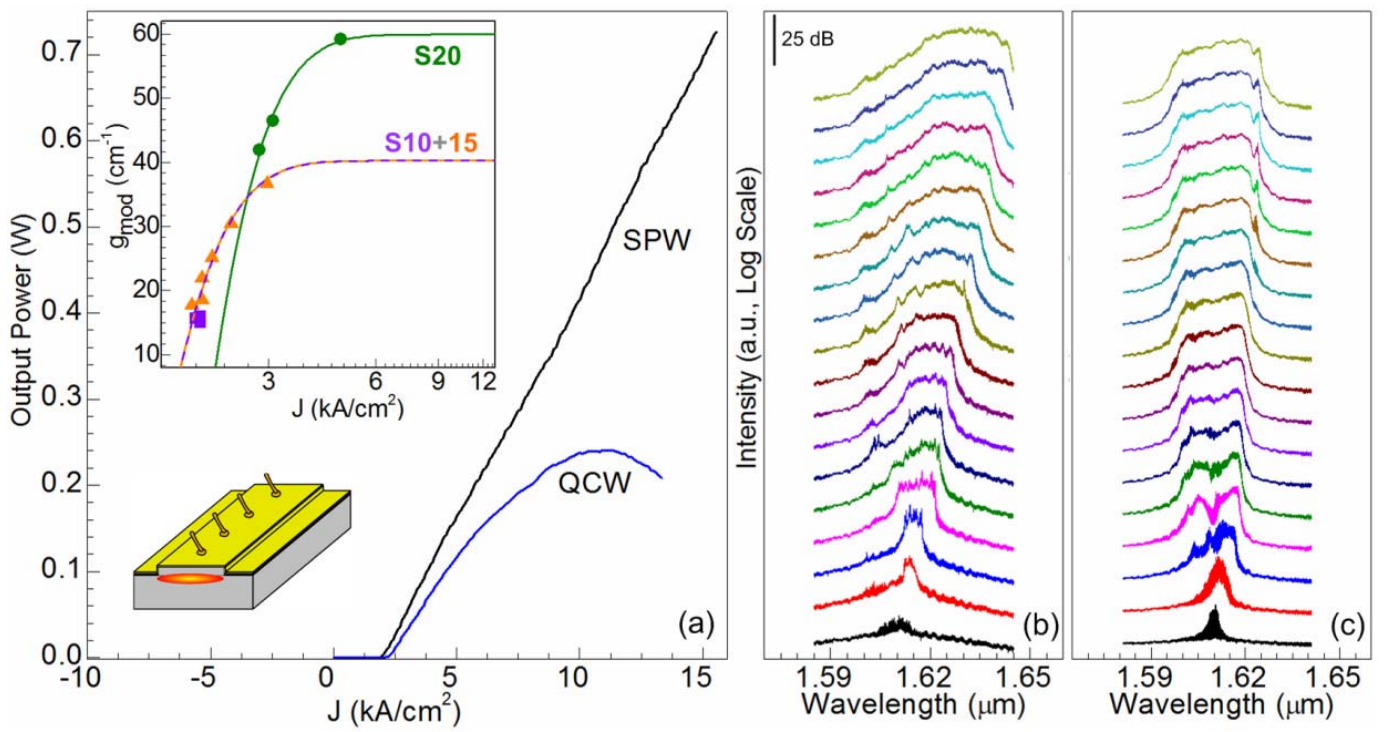

Fig. 7. (a) Room temperature $L-I$ characteristics of $15 \times 1600 \mu m$ long CQD oxide-strip laser at different pulsed operation. The corresponding progressive lasing spectra with increasing injection current density in the (b) QCW and (c) SPW operation. The inset of (a) shows the modal gain of the $W=15 \mu m$ versus the threshold current density, obtained with devices of different cavity lengths $(L=0.25-3.0 \mathrm{~mm})$. The ideality factor and internal loss are fixed at $\gamma=3$ and $\alpha_{i}=11 \mathrm{~cm}^{-1}$, respectively. In all the cases, the injection current density is varied from $1.0 J_{t h}, 1.1 J_{t h}$, and $1.25 J_{t h}$ to $5.0 J_{t h}$ in steps of $0.25 J_{t h}$. Similar lasing operation is observed from all the cavity lengths tested in the both the operation.

from the central transition energy of the middle $\mathrm{S}_{15}$ dash stack once compensating for the total loss of the system (optical loss and $\alpha_{i}$ ). In fact, the lasing linewidth obtained from these cavities exceed the ever reported lasing bandwidth utilizing Qdash nano-structure active region, by $\sim 20 \%$, as shown in the inset of Fig. 6(c) [3], [4], [23]. We ascribe this enhanced broad emission to the non-localized inhomogeneity across the Qdash stacks i.e. carrier access to the either side of the widely distributed Qdash optical-transition energies (with overlapping DOS tails). In other words, availability of both $S_{20}$ and $S_{15}$ dash ensembles to fill up by carriers on increasing current injection, thus, leasing to simultaneous lasing from all the dash stacks. In the case of long (short) cavities, the carriers are probably restricted by fewer DOS at lower (higher) dash optical-transition energies owing to their onset of lasing from the $S_{10}\left(S_{20}\right)$ dash stacks, thus hindering to exploit the complete inhomogeneity of the active region.

Three distinct emission humps in the SPW mode lasing spectra has been observed in all the cavity length lasers $(L>0.45 \mathrm{~nm})$ under high current injection, emerging from the three Qdash groups [Fig. 6(b)]. On progressive increase in the pumping current, appreciable overlap of the $S_{15}$ and $S_{10}$ emission lobes is observed in all the cavity lengths, suggesting uniform distribution of dash electronic states among these highly inhomogeneous stacks. However, an imprecise overlap of $S_{20}$ spectral lobe with some distinct spectral modulations and visible Fabry-Perot resonances, even at high injections, demonstrates some photon re-absorption process occurring in the system. This can be assessed by observing the IEL of each dash ensemble separately, for the $L=1.6 \mathrm{~mm}$ laser cavity, as a function of current injection, shown in Fig. 6(c). We followed the emission boundaries discussed earlier to obtain these curves. As seen from Figs. 2(b) and 6(c), when the current pumping is $<2.4 J_{t h}$, the lasing emission is dominated by the $\mathrm{S}_{15}$ dash ensembles, which progressively broadening with further pumping. This is due to simultaneously lasing of different in-plane dispersive dashes of $\mathrm{S}_{15}$ and partially from the $S_{10}$ dashes which are reaching population inversion. This continues until $\sim 2.5 J_{t h}$ beyond which $S_{15}$ dashes tend to saturate (reaching the gain saturation), and onset of lasing from $S_{10}$ and later from $S_{20}$ dash ensembles [see Fig. 2(b)] is observed. A sharp increase in the slope of IEL curves of both the dash groups is an indication of the beginning of the stimulated emission. Note that because of this pulsed operation being carrier sufficient system (with no appreciable spill-over of carriers), $S_{15}$ dashes would be able to maintain the rate of photon generation and absorption and therefore no quenching of the middle wavelength longitudinal modes. Now, the carriers are more effectively captured by the other dash groups and the IEL indicates that $S_{10}$ eventually dominates the lasing emission and competes with $\mathrm{S}_{15}$ dash groups at $>3.5 J_{t h}$ [overlap of the respective emission lobes seen in Fig. 2(b)]. In general, in a Qdash system with dispersive geometries, population inversion preferentially occurs in small average height $S_{20}$ dashes acquiring dot-like features (tight lateral confinement, lower modal gain and DOS) that require smaller number of carriers. The generated high-energy photons from $S_{20}$ dashes gets absorbed by large average height $S_{15}$ dashes (weak lateral confinement, higher modal gain and DOS) [31]. With increasing pumping, this carrier feeding mechanism shifts to the $S_{15}$ and $S_{10}$ dash ensembles. In other words, $S_{20}\left(S_{15}\right)$ dashes behave as carrier feeders to $S_{15}\left(S_{20}\right)$ dashes at low (high) current injections. With increasing current injection, it is possible for all the three dash ensembles to sustain lasing and broadens in spite of not so severe photon re-absorption in the system, leading to an ultra broad emission from this chirped active region device. 


\section{B. Broad Area Lasers}

The behavior of Qdash gain media in the broad area lasers is understood by referring Fig. 7 which correspond to a $15 \times 1600 \mu \mathrm{m}$ long CQD laser diode. In the SPW mode [Fig. 7(c)], a typical broadening of lasing spectrum along with a smooth $L-I$ curve is observed. Fig. 7(b) shows an appreciable red shift in the lasing spectra of the QCW mode and an early roll-off of the $L-I$ curve dictating excessive device heating in this case due to the large $W$ (increase in laser geometrical parameters, particularly $W$ ). Nevertheless, a single broad linewidth laser emission in both the pulsed operations [Fig. 7(b) and (c)], at intermediate injection, suggests that multiple and dissimilar wavelength emission from different Qdash ensembles is inhibited in this case, probably due to phonon assisted carrier tunneling, as discussed earlier. To understand the cause of this observation, we analyzed the gain profiles of the active region utilizing the cavity length threshold current density dependence of the modal gain [see inset of Fig. 7(a)]. The $\eta_{i}$ and $\alpha_{i}$, obtained in this case from different cavities, is similar in value $\left(\sim 85 \%\right.$ and $11 \mathrm{~cm}^{-1}$, respectively) to that obtained in the ridge-waveguide lasers. Again, two gain curves are observed in this case, on fitting the gain expression (Eqn. 1) with the experimental data of SPW pulsed operation and QCW operation (not shown). The obtained values of $J_{t r}\left(g_{s a t}\right)$ of the $\mathrm{S}_{10+15}$ and $\mathrm{S}_{20}$ dash ensembles in the SPW mode are $1.6 \mathrm{kA} / \mathrm{cm}^{2}\left(40.0 \mathrm{~cm}^{-1}\right)$ and $2.0 \mathrm{kA} / \mathrm{cm}^{2}\left(60.0 \mathrm{~cm}^{-1}\right)$, respectively. These values are in good agreement with the different ridge-width lasers results, showing the consistency of our analysis. Moreover, observation of two peak lasing spectra at near threshold current values, in both the pulsed operation (sharp in the SPW mode and weak in the QCW mode), further validate our attribution of two dash groups governing the lasing operation. Eventually, a single broad emission is achieved at high injection suggesting simultaneous recombination of carriers from somewhat uniformly distributed dash states among both the dash families, attaining a lasing bandwidth of $\sim 20 \mathrm{~nm}$ in the SPW mode. Therefore, our detailed analysis of CQD lasers under different optical wave guiding schemes show that for $W>4 \mu \mathrm{m}$, the active medium is dominated by only two groups of Qdashes which limits the inhomogeneous broadening of the active region and eventually the lasing bandwidth.

\section{CONCLUSION}

In summary, we demonstrated the enhancement in the active region inhomogeneity of InAs/InGaAlAs/InP quantumdash-in-well structure, through utilization of variable barrier thickness InGaAlAs barrier in the multi-stack Qdash structure. A broad stimulated emission linewidth of $>50 \mathrm{~nm}$ is obtained at room temperature from the $2 \times 830 \mu \mathrm{m}$ laser device, under the SPW operation. The effect of ridge-width and different waveguide mechanisms, on the lasing operation under both SPW and QCW mode, highlighted the very different behavior of dash active region, resulting in one being a carrier sufficient system and other carrier deficient system, in general. Our work elucidates the device physics of chirped Qdash laser structure, particularly the optically pumped carrier feeding mechanism among the dash ensembles, and help in further optimizing the multi-stack Qdash active region. Moreover, our results suggest that the chirped InAs/InGaAlAs Qdash structure with enhanced broad gain profile forms a viable platform for the generation of a novel broadband semiconductor laser source with potential ultra-broad emission through monolithic integration of multiple bandgap sections using post growth intermixing techniques.

\section{REFERENCES}

[1] B. S. Ooi, H. S. Djie, Y. Wang, C. L. Tan, J. C. M. Hwang, X. M. Fang, et al., "Quantum dashes on InP substrate for broadband emitter applications," IEEE J. Sel. Topics Quantum Electron., vol. 14, no. 4, pp. 1230-1238, Jul./Aug. 2008.

[2] Z. Y. Zhang, R. A. Hogg, X. Q. Lv, and Z. G. Wang, "Self-assembled quantum-dot superluminescent light-emitting diodes," Adv. Opt. Photon., vol. 2, no. 2, pp. 201-228, 2010.

[3] H. S. Djie, B. S. Ooi, X. M. Fang, Y. Wu, J. M. Fastenau, W. K. Liu, et al., "Room-temperature broadband emission of an InGaAs/GaAs quantum dots laser," Opt. Lett., vol. 32, no. 1, pp. 44-46, 2007.

[4] H. S. Djie, C. L. Tan, B. S. Ooi, J. C. M. Hwang, X. M. Fang, Y. Wu, et al., "Ultrabroad stimulated emission from quantum-dash laser," Appl. Phys. Lett., vol. 91, pp. 111116-1-111116-3, 2007.

[5] C. Gmachl, D. L. Sivco, R. Colombelli, F. Capasso, and A. Y. Cho, "Ultra-broadband semiconductor laser," Nature, vol. 415 , pp. 883-887, Feb. 2002

[6] C. L. Tan, H. S. Djie, and B. S. Ooi, "Novel multiwavelength emitter for WDM transmission utilizing broadband quantum-dash laser diode," in Proc. CLEO, Jun. 2009, pp. 1-2.

[7] C.-S. Lee, W. Guo, D. Basu, and P. Bhattacharya, "High performance tunnel injection quantum dot comb laser," Appl. Phys. Lett., vol. 96, pp. 101107-1-101107-3, Mar. 2010.

[8] H. S. Djie, C. E. Dimas, and B. S. Ooi, "Wideband quantum-dash-inwell superluminescent diode at $1.6 \mu \mathrm{m}, "$ IEEE Photon. Technol. Lett., vol. 18, no. 16, pp. 1747-1749, Aug. 15, 2006.

[9] H. S. Djie, C. E. Dimas, D. N. Wang, B. S. Ooi, J. C. Hwang, G. T. Dang, et al., "InGaAs/GaAs quantum-dot superluminescent diode for optical sensor and imaging," IEEE Sensors J., vol. 7, no. 2, pp. 251-257, Feb. 2007.

[10] K. J. Zhou, Q. Jiang, Z. Y. Zhang, S. M. Chen, H. Y. Liu, Z. H. Lu, et al., "Quantum dot selective area intermixing for broadband light sources," Opt. Exp., vol. 20, no. 24, pp. 26950-26957, 2012.

[11] C. L. Tan, H. S. Djie, Y. Wang, C. E. Dimas, V. Hongpinyo, Y. H. Ding, et al., "Wavelength tuning and emission width widening of ultrabroad quantum dash interband laser," Appl. Phys. Lett., vol. 93, pp. 111101-1-111101-3, Sep. 2008.

[12] A. Kovsh, I. Krestnikov, D. Livshits, S. Mikhrin, J. Weimert, and A. Zhukov, "Quantum dot laser with $75 \mathrm{~nm}$ broad spectrum of emission," Opt. Lett., vol. 32, no. 7, pp. 793-795, 2007.

[13] L. Li, M. Rossetti, A. Fiore, L. Occhi, and C. Vélez, "Wide emission spectrum from superluminescent diodes with chirped quantum dot multilayers," Electron. Lett., vol. 41, no. 1, pp. 41-43, 2005.

[14] S. Haffouz, P. J. Barrios, R. Normandin, D. Poitras, and Z. Lu, "Ultrawide-bandwidth, superluminescent light-emitting diodes using InAs quantum dots of tuned height," Opt. Lett., vol. 37, no. 6, pp. 1103-1105, 2012.

[15] J. X. Chen, A. Markus, A. Fiore, U. Oesterle, R. P. Stanley, J. F. Carlin, et al., "Tuning InAs/GaAs quantum dot properties under StranskiKrastanov growth mode for $1.3 \mu \mathrm{m}$ applications," J. Appl. Phys., vol. 91, no. 10 , pp. $6710-6716,2002$.

[16] P. D. Judson, K. M. Groom, D. T. Childs, M. Hopkinson, N. Krstajic, and R. A. Hogg, "Maximising performance of optical coherence tomography systems using a multi-section chirped quantum dot superluminescent diode," Microelectron. J., vol. 40, no. 3, pp. 588-591, 2009.

[17] S. M. Chen, K. J. Zhou, Z. Y. Zhang, D. T. D. Childs, M. Hugues, A. J. Ramsay, et al., "Ultra-broad spontaneous emission and modal gain spectrum from a hybrid quantum well/quantum dot laser structure," Appl. Phys. Lett., vol. 100, no. 4, pp. 041118-1-041118-3, 2012.

[18] K. Nishi, H. Saito, S. Sugou, and J. S. Lee, "A narrow photoluminescence linewidth of $21 \mathrm{meV}$ at $1.35 \mu \mathrm{m}$ from strain-reduced InAs quantum dots covered by $\operatorname{In}_{0.2} \mathrm{Ga}_{0.8}$ As grown on GaAs substrates," Appl. Phys. Lett., vol. 74, no. 8, pp. 1111-1113, 1999. 
[19] A. Sauerwald, T. Kummell, G. Bacher, A. Somers, R. Schwertberger, J. P. Reithmaier, et al., "Size control of InAs quantum dashes," Appl. Phys. Lett., vol. 86, pp. 253112-1-253112-3, Jun. 2005.

[20] P. Miska, J. Even, C. Paranthoen, O. Dehaese, A. Jbeli, M. Senes, et al., "Vertical electronic coupling between InAs/InP quantum-dot layers emitting in the near-infrared range," Appl. Phys. Lett., vol. 86, pp. 111905-1-111905-3, Mar. 2005.

[21] C. L. Tan, H. S. Djie, Y. Wang, C. E. Dimas, V. Hongpinyo, Y. H. Ding, et al., "The influence of nonequilibrium distribution on room-temperature lasing spectra in quantum-dash lasers," IEEE Photon. Technol. Lett., vol. 21, no. 1, pp. 30-32, Jan. 1, 2009.

[22] A. E. Zhukov, M. V. Maksimov, and A. R. Kovsh, "Device characteristics of long-wavelength lasers based on self-organized quantum dots," Semiconductors, vol. 46, no. 10, pp. 1225-1250, 2012.

[23] M. Z. M. Khan, T. K. Ng, C.-S. Lee, P. Bhattacharya, and B. S. Ooi, "Chirped InAs/InP quantum-dash laser with enhanced broad spectrum of stimulated emission," Appl. Phys. Lett., vol. 102, no. 9, pp. 091102-1-091102-3, 2013.

[24] M. Z. M. Khan, T. K. Ng, C.-S. Lee, P. Bhattacharya, and B. S. Ooi, "Distinct lasing operation from chirped InAs/InP quantum-dash laser," IEEE Photon. J., vol. 5, no. 4, p. 1501308, Aug. 2013.

[25] M. Achtenhagen, A. A. Hardy, and C. S. Harder, "Coherent kinks in high-power ridge waveguide laser diodes," J. Lightw. Technol., vol. 24, no. 5, pp. 2225-2232, May 2006

[26] Q. Cao, S. F. Yoon, C. Y. Liu, and C. Y. Ngo, "Narrow ridge waveguide high power single mode $1.3-\mu \mathrm{m}$ InAs/InGaAs ten-layer quantum dot lasers," Nanoscale Res. Lett., vol. 2, pp. 303-307, Jun. 2007.

[27] M. L. Xu, G. L. Tan, R. Clayton, and J. M. Xu, "Increased threshold for the first-order lateral mode lasing in low-ridge waveguide high power QW lasers," IEEE Photon. Technol. Lett., vol. 8, no. 11, pp. 1444-1446, Nov. 1996

[28] S. G. Li, Q. Gong, Y. F. Lao, H. D. Yang, S. Gao, P. Chen, et al., "Twocolor quantum dot laser with tunable wavelength gap," Appl. Phys. Lett., vol. 95, pp. 251111-1-251111-3, Dec. 2009.

[29] M. Z. M. Khan, T. K. Ng, U. Schwingenschlogl, P. Bhattacharya, and B. S. Ooi, "Effect of the number of stacking layers on the characteristics of quantum-dash lasers," Opt. Exp., vol. 19, no. 14, pp. 13378-13385, 2011.

[30] P. Podemski, R. Kudrawiec, J. Misiewicz, A. Somers, R. Schwertberger, J. P. Reithmaier, et al., "Thermal quenching of photoluminescence from InAs $/ \mathrm{In}_{0.53} \mathrm{Ga}_{0.23} \mathrm{Al}_{0.24} \mathrm{As} / \mathrm{In} P$ quantum dashes with different sizes," Appl. Phys. Lett., vol. 89, pp. 151902-1-151902-3, Oct. 2006.

[31] M. Z. M. Khan, T. K. Ng, U. Schwingenschlogl, P. Bhattacharya, and B. S. Ooi, "Modeling the lasing spectra of InAs/InP quantum dash lasers," Appl. Phys. Lett., vol. 98, pp. 101105-1-101105-3, Mar. 2011.

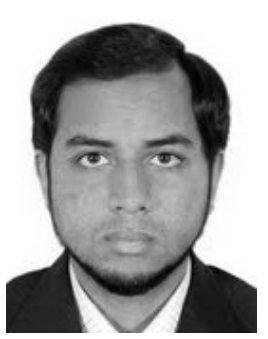

Mohammed Zahed Mustafa Khan received the B.E. in Electronics and Communication from Osmania University, India, in 2001, and the MS in Electrical Engineering from King Fahd University of Petroleum and Minerals (KFUPM), Saudi Arabia, in 2004. From 2004-2009, he was a lecturer at KFUPM, Hafr-Al-Batin campus, Saudi Arabia.

Currently, he is with Photonics Laboratory, King Abdullah University of Science and Technology (KAUST), Saudi Arabia, where he is pursuing Ph.D. in the field of Photonics, particularly, focusing on the theoretical and experimental work on quantum confined nano-structure lasers particularly on broadband quantum-dash lasers.

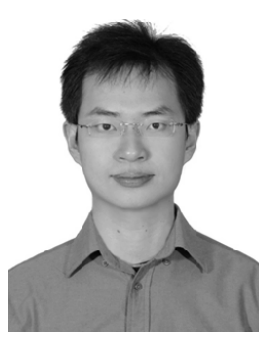

Chi-Sen Lee received the B.S. degree in electrical engineering from National Taiwan Ocean University, Keelung, Taiwan, the M.S. degree in electronics engineering from National Taiwan University, Taipei, Taiwan, and the Ph.D. degree in electrical engineering from the University of Michigan, Ann Arbor, in 2003, 2005, and 2011, respectively. $\mathrm{He}$ is currently a Device Design Engineer with Avago Technologies, Breinigsville, PA, USA, where he is engaged in research on the design and testing of XFP Tunable TOSA. His current research interest include III-V semiconductor diode lasers, silicon photonic-integrated devices, and both monolithic and hybrid integration techniques on III-V and silicon integration for optoelectronic devices.

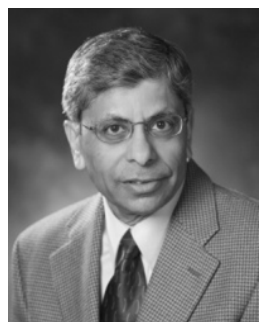

Pallab Bhattacharya is the Charles M. Vest Distinguished University Professor of electrical engineering and computer science and the James R. Mellor Professor of engineering with the Department of Electrical Engineering and Computer Science, University of Michigan, Ann Arbor. He received the M.Eng. and Ph.D. degrees from the University of Sheffield, U.K., in 1976 and 1978, respectively. He was an Editor of the IEEE TRANSACTIONS ON ELECTRON DEVICES and an Editor-in-Chief of the Journal of Physics D. He has edited Properties of Lattice-Matched and Strained InGaAs (U.K.: INSPEC, 1993) and Properties of III-V Quantum Wells and Superlattices (U.K.: INSPEC, 1996). He has authored the textbook Semiconductor Optoelectronic Devices (Prentice Hall). His teaching and research interests are in the areas of compound semiconductors, low-dimensional quantum confined systems, nanophotonics, spintronics, and optoelectronic integrated circuits. He is currently working on high-speed quantum dot lasers, nitride-based visible quantum dot lasers and LEDs, nanowire heterostructures, cavity quantum electrodynamics, and polariton lasers.

$\mathrm{He}$ is a member of the National Academy of Engineering. He has received the John Simon Guggenheim Fellowship, the Heinrich Welker Medal, the IEEE (EDS) Paul Rappaport Award, the IEEE (LEOS) Engineering Achievement Award, the IEEE (Nanotechnology Council) Nanotechnology Pioneer Award, the Optical Society of America (OSA) Nick Holonyak Award, the TMS John Bardeen Award, the SPIE Technical Achievement Award, and the Quantum Devices Award of the International Symposium on Compound Semiconductors. He has received the S.S. Attwood Award, the Kennedy Family Research Excellence Award, and the Distinguished Faculty Achievement Award from the University of Michigan. He is a fellow of the American Physical Society, the Institute of Physics, U.K., and the OSA.

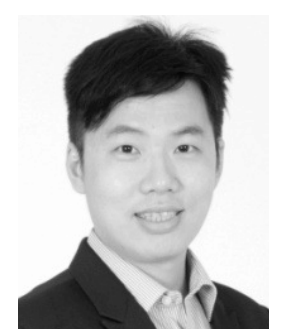

Tien Khee Ng received his Ph.D. and M.Eng. in electrical and electronics engineering from Nanyang Technological University (NTU), Singapore, in 2005 and 2001 , respectively. He was a member of technical staff with Tinggi Technologies, a high brightness blue LED start-up company at Science-ParkI, Singapore, from 2004-2006, and later a research fellow at NTU until 2009. He is currently a research scientist at King Abdullah University of Science and Technology (KAUST), Saudi Arabia, and the coprincipal investigator for the KACST's technology innovation center for solid-state lighting at KAUST.

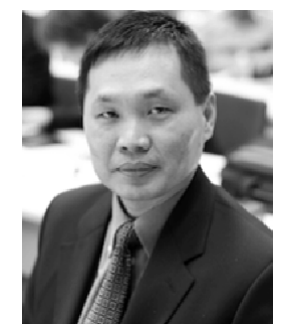

Boon S. Ooi (M'95-SM'03) received the Ph.D. degree in electronics and electrical engineering from the University of Glasgow, U.K. He served as a Faculty Member with Nanyang Technological University, Singapore, and Lehigh University, USA, before he joined the King Abdullah University of Science and Technology as a Professor of electrical engineering in 2009. His research interests include semiconductor lasers and photonics integrated circuits. He is a fellow of the SPIE and the Institute of Physics, U.K. 